\title{
Uso de $n$-alcanos na estimativa da composição botânica da dieta em ovinos alimentados com diferentes proporções de Brachiaria decumbens Stapf e Arachis pintoi Koprov e Gregory ${ }^{1}$
}

\section{Nelson Massaru Fukumoto ${ }^{2}$, Julio Cesar Damasceno ${ }^{3}$, Cristiano Côrtes $^{4}$, Lauri Roehsig 5 , Fabíola Cristine de Almeida Rego ${ }^{4}$, Ulysses Cecato ${ }^{3}$, Antonio Ferriani Branco ${ }^{3}$}

\author{
${ }^{1}$ Parte da dissertação de Mestrado do primeiro autor apresentada à Universidade Estadual de Maringá (UEM). Pesquisa financiada pelo \\ CNPq. \\ 2 Doutorando em Zootecnia da UEM. \\ ${ }^{3}$ Departamento de Zootecnia - UEM, Maringá - PR. \\ ${ }^{4}$ Doutor em Zootecnia pela UEM. \\ ${ }^{5}$ Mestre em Zootecnia pela UEM.
}

RESUMO - Neste experimento objetivou-se avaliar o poder discriminatório dos $n$-alcanos para estimar com acurácia e precisão a composição botânica da dieta em ovinos alimentados com diferentes proporções de Arachis pintoi Koprov \& Gregory cv. Amarillo (0, 15, 30, 45 e 60\%) e Brachiaria decumbens Stapf. Foram utilizados 20 ovinos em delineamento inteiramente casualizado, com período experimental de dez dias de adaptação à dieta e cinco dias de coleta de fezes. Nas amostras (compostas) de fezes do período e nos fenos, foi analisada a concentração de $n$-alcanos. Para o cálculo da composição botânica, utilizou-se minimização da soma dos quadrados dos desvios, considerando as concentrações dos alcanos nos componentes da dieta e nas fezes. Para a escolha dos alcanos mais discriminatórios, foram utilizadas as análises multivariadas e as variáveis canônicas. As estimativas calculadas foram submetidas à análise de variância. As médias foram comparadas pelo teste $\mathrm{t}$ e as correções dos valores estimados em relação aos valores reais foram ajustadas em regressão linear As variáveis canônicas indicaram que os alcanos $C_{35}, C_{33}, C_{30}, C_{31}, C_{27}, C_{29}$ e $C_{36}$ são os de maior potencial discriminatório. $\mathrm{O}$ uso desses alcanos nos cálculos foi mais acurado e preciso para estimar a proporção de A. pintoi na dieta que o uso de apenas dois ou três alcanos com poder discriminatório. O melhor ajuste da regressão também foi encontrado para esses alcanos $\mathrm{O}$ teste $\mathrm{t}$ para o intercepto da equação (a) e o coeficiente de regressão (b) indicaram que $\mathrm{a}=0$ e $\mathrm{b}=1$, comprovando que os valores estimados são equivalentes aos valores reais. As análises multivariadas mostraram-se ferramentas de grande importância na escolha dos $n$-alcanos nos cálculos nas estimativas.

Palavras-chave: análises multivariadas, cera cuticular, consorciação de pastagens, indicadores, seletividade alimentar

\section{Use of $\boldsymbol{n}$-alkanes to estimate the dietary botanical composition in sheep fed different proportions of Brachiaria decumbens Stapf and Arachis pintoi Koprov and Gregory}

\footnotetext{
ABSTRACT - The objective of this experiment was to use $n$-alkane to estimate accurately and precisely the botanical composition of dietary forage in sheep fed different proportions of Arachis pintoi Koprov \& Gregory cv. Amarillo and Brachiaria decumbens Stapf $(0,15,30,45$ e $60 \%$ of Arachis pintoi). Twenty lambs were used in a completely randomized design with 10 days for diet adaptation and 5 days for fecal collection. Both fecal composites and hay samples were analyzed for $n$-alkane concentration. Dietary forage proportions were estimated from measured concentrations of $n$-alkanes in feces and forage by the least square sum of deviations. Multivariate analyses and canonical variables were used to select the most discriminatory alkane with the obtained data submitted to analysis of variance. Means were compared using the " $\mathrm{t}$ " test and the adjustments of the estimated values in relation to the observed were done by linear regression. The canonical variables indicated that the alkanes $\mathrm{C}_{35}, \mathrm{C}_{33}, \mathrm{C}_{30}, \mathrm{C}_{31}, \mathrm{C}_{27}, \mathrm{C}_{29}$ and $\mathrm{C}_{36}$ had the greatest discriminatory potential. Therefore, the use of all these alkanes in the calculation was more accurate and precise $(\mathrm{P}<0.05)$ than the use of a combination of 2 or 3 alkanes to estimate the proportion of $A$. pintoi in the diet. The best adjustment of the regression was also found for these alkanes. The " $\mathrm{t}$ " test for the intercept of the equation (a) and the regression coefficient (b) showed that $\mathrm{a}=0$ and $\mathrm{b}=1$, indicating that the estimated values are equivalent to the observed. The multivariate analysis appears to be an important tool to select $n$-alkanes for diet evaluation.
}

Key Words: cuticular wax, feed selection, herbage mixtures pasture, markers, multivariate analysis 


\section{Introdução}

A composição de espécies forrageiras disponíveis para o pastejo é uma característica importante nos estudos nutricionais, especialmente em pastagens consorciadas que possam proporcionar melhor desempenho animal com a presença de leguminosas ou em pastagens com maior proporção de leguminosas (Dove, 1992).

Como relatado por Chen et al. (1999), a composição da forragem consumida pode ser tão importante quanto a quantidade consumida. Portanto, a alta ingestão de determinadas espécies ou frações de plantas não tem efeito apenas no aporte nutricional dos animais; tem importante influência sobre a sustentabilidade do sistema solo $\times$ planta $\times$ animal.

Estudos sobre a presença da leguminosa em pastagens consorciadas com gramíneas fornecem dados sobre os efeitos dessa gramínea no animal. Entretanto, não se sabe quali e quantitativamente a quantidade de leguminosa, ou que parte dela, é consumida no processo de seleção.

Métodos convencionais para estimar o consumo e a composição botânica da dieta de animais em pastejo, como a observação direta das espécies e das partes consumidas pelo animal, o uso de fístulas esofagianas e o exame do conteúdo estomacal, são técnicas pouco precisas ou de difícil execução e que interferem no comportamento de pastejo dos animais (Dove \& Mayes, 1991).

Entre as metodologias existentes, Dove \& Mayes (1999) destacaram o uso dos indicadores naturais presentes nas plantas, os $n$-alcanos, pois não prejudicam a saúde dos animais ou do homem e possibilitam estimativas acuradas da ingestão de MS e da composição botânica das dietas consumidas pelos animais.

Cada planta possui, teoricamente, perfil único de $n$-alcanos, que, por meio de sistemas de equações ou métodos interativos de cálculos, permite estimar a composição de misturas complexas, uma vez que, potencialmente, os $n$-alcanos poderiam discriminar 10 a 15 componentes de uma dieta, em razão do grande número desses hidrocar-bonetos com potencial discriminatório (Dove \& Mayes, 1996).

Entretanto, nem todos os $n$-alcanos contribuem de forma efetiva na discriminação dos componentes dietéticos e alguns ainda adicionam importantes fontes de erro às estimativas (Dove \& Mayes, 1999; Côrtes et al., 2005). Neste contexto, estudos que gerem informações sobre a identificação dos $n$-alcanos mais adequados para utilização nos cálculos e obtenção de estimativas acuradas de composição da dieta permitem detectar diferenças na seletividade de animais em pastejo.
Este trabalho foi realizado com os objetivos de avaliar a acurácia e a precisão da técnica de $n$-alcanos para estimativa da composição da dieta com diferentes níveis de Brachiaria decumbens e Arachis pintoi em ovinos e identificar a combinação de $n$-alcanos com uso de análises multivariadas que permitam estimar as proporções dos componentes dietéticos com maior precisão.

\section{Material e Métodos}

O experimento foi realizado no setor de Ovinocultura e Caprinocultura da Fazenda Experimental de Iguatemi (FEI) da Universidade Estadual de Maringá (UEM/Maringá-PR).

Foram utilizados 20 ovinos machos, sem raça definida, com 24,59 $\pm 2,78 \mathrm{~kg}$ de PV, em delineamento experimental inteiramente casualizado, com cinco tratamentos (dietas) e quatro repetições. As dietas fornecidas aos animais foram diferentes proporções de fenos de Arachis pintoi Koprov \& Gregory cv. Amarillo e Brachiaria decumbens Stapf $(0,15,30,45$ e $60 \%$ de Arachis pintoi). O feno de Arachis pintoi foi confeccionado no Instituto Agronômico do Paraná (IAPAR - Estação Experimental de Londrina) e o de Brachiaria decumbens, no Campus da Universidade Estadual de Maringá (UEM). Os materiais foram picados grosseiramente e posteriormente misturados e homogeneizados em diferentes proporções para compor as dietas experimentais.

Os animais foram alojados em baias individuais (piso ripado e suspenso) com livre acesso ao bebedouro e ao cocho, onde receberam sal mineral à vontade. No início do experimento, todos os animais foram vermifugados.

O período experimental foi de 15 dias, dez de adaptação dos animais à dieta e cinco de coleta de fezes. Durante o período experimental, os animais foram alimentados duas vezes ao dia (às 8 e 16h). No período de adaptação, os animais receberam feno à vontade, permitindo-se sobra de aproximadamente $15 \%$. No período de coleta de fezes, os animais receberam alimentação restrita $(90 \%$ do consumo voluntário) para evitar sobras. A dieta fornecida foi devidamente pesada por animal e por dia.

Para a coleta total de fezes, os animais foram equipados com bolsas coletoras adaptadas ao dorso do animal. A produção total de fezes foi quantificada diariamente por animal.

Durante o período de coleta de fezes, também foram amostrados diariamente os fenos fornecidos. As amostras de fezes e de fenos coletadas diariamente foram armazenadas em freezer $\left(-10^{\circ} \mathrm{C}\right) \mathrm{e}$, posteriormente, foram secas em estufa de ventilação forçada $\left(55^{\circ} \mathrm{C}\right.$ por 72 horas $)$ e trituradas em moinho com peneiras com crivos de $1 \mathrm{~mm}$. Depois de 
moídas, formaram amostras compostas, dos cinco dias de coleta, com base na MS (100 g de fezes/animal/dia). O mesmo foi aplicado às amostras de feno.

A extração dos $n$-alcanos foi realizada no Laboratório de Nutrição Animal do Departamento de Zootecnia (DZO) da UEM.

Para análise de $n$-alcanos, utilizou-se a técnica desenvolvida por Vulich et al. (1995) modificada, que se baseia no processo de saponificação direta das amostras. Foram pesadas em balança analítica amostras de feno $(1,5 \mathrm{~g})$ e de fezes moídas $(0,5 \mathrm{~g})$ utilizando peneira com crivos de $1 \mathrm{~mm}$. Nos frascos de $50 \mathrm{~mL}$, com tampa de rosca, foram adicionados 0,3016 mg de tetratriacontano $99,7 \%\left(\mathrm{C}_{34} \mathrm{H}_{70}-\right.$ Sigma Aldrich) diluídos em $4 \mathrm{~mL}$ de $n$-heptano $95 \%\left(\mathrm{C}_{7} \mathrm{H}_{16}\right)$ a cada amostra como padrão interno. Evaporado o $n$-heptano, acrescentaram-se $14 \mathrm{~mL}$ de solução alcoólica de hidróxido de potássio $(\mathrm{KOH}$ a 1,5 M) e procedeu-se à incubação em banho-maria a $90^{\circ} \mathrm{C}$, sob agitação durante 4,5 horas. Após resfriamento da amostra à temperatura ambiente, foram adicionados $20 \mathrm{~mL}$ de heptano e $10 \mathrm{~mL}$ de água destilada, mantendo a mistura a $60^{\circ} \mathrm{C}$, sob agitação vigorosa, durante 15 minutos. Após a decantação (aproximadamente 1 hora), o sobrenadante foi coletado com uma pipeta de pasteur em tubos de ensaio e parcialmente evaporado. Em seguida, o conteúdo foi purificado em colunas de sílica-gel (200 - 400 mesh - Acros Organics) e suspenso em heptano, com volume de leito de $10 \mathrm{~mL}$. A mistura heptano contendo $n$-alcanos foi recuperada em balões de $50 \mathrm{~mL}$, sendo evaporado todo heptano em temperatura ambiente, mantendo somente os hidrocarbonetos sólidos aderidos. Posteriormente, os $n$-alcanos foram rediluídos em microtubos de $2 \mathrm{~mL}$ com $1,5 \mathrm{~mL}$ de $n$-heptano $95 \%\left(\mathrm{C}_{7} \mathrm{H}_{16}\right)$.

A identificação dos $n$-alcanos das amostras foi realizada por cromatografia gasosa no Laboratório de Alimentos do Departamento de Química (DQI) da UEM.

Foi utilizado cromatógrafo a gás SHIMADZU 14 com coluna capilar de sílica fundida (OV-5 de $30 \mathrm{~m}$ x 0,32 mm e $0,25 \mu \mathrm{m}$ de espessura de filme) e detector de ionização de chama (FID). Os fluxos dos gases ultrapuros (White Martins) foram de $1,2 \mathrm{~mL} / \mathrm{min}$ para o gás de arraste $\left(\mathrm{H}_{2}\right)$ e $30 \mathrm{~mL} / \mathrm{min}$ para o gás auxiliar (Make-up) $\left(\mathrm{N}_{2}\right)$ e 30 e $300 \mathrm{~mL} / \mathrm{min}$ para os gases de chama $\left(\mathrm{H}_{2}\right.$ e ar sintético, respectivamente).

As temperaturas do injetor (INLET) e do detector (FID) foram $300 \mathrm{e} 315^{\circ} \mathrm{C}$, respectivamente. A coluna foi operada com temperatura inicial de $220^{\circ} \mathrm{C}$ por 2 minutos, com aumento na razão de $5^{\circ} \mathrm{C} /$ minuto até $297^{\circ} \mathrm{C}$. O modo de injeção foi Split (1:100) e a amostra $(3,0 \mu \mathrm{L})$ foi injetada manualmente com microseringa de $10 \mu \mathrm{L}$.

As áreas dos picos cromatográficos correspondentes a cada $n$-alcano foram determinadas por meio de um
Integrador-Processador CG-300, no qual a identificação dos $n$-alcanos de comprimento de cadeia $\mathrm{C}_{24}$ a $\mathrm{C}_{36}$ se baseou na comparação com padrão (Acros Organics), pelo tempo de retenção médio, contendo os alcanos tetracosano $\left(\mathrm{C}_{24} \mathrm{H}_{50}\right)$, hexacosano $\left(\mathrm{C}_{26} \mathrm{H}_{54}\right)$, dotriacontano $\left(\mathrm{C}_{32} \mathrm{H}_{66}\right)$, tetratriacontano $\left(\mathrm{C}_{34} \mathrm{H}_{70}\right)$ e hexatriacontano $\left(\mathrm{C}_{36} \mathrm{H}_{74}\right)$. Posteriormente, foram convertidas as quantidades de $n$-alcanos por referência ao padrão interno $\mathrm{C}_{34} \mathrm{H}_{66}$ (0,3016 mg/amostra) e calculados por quilograma de MS para a amostra considerada.

As correções das concentrações dos alcanos $\mathrm{C}_{24}$ a $\mathrm{C}_{36}$ nas fezes foram realizadas utilizando-se os valores de recuperação fecal obtidos no período de estimação do consumo e da digestibilidade.

Para o cálculo da composição botânica, utilizou-se a equação proposta por Mayes et al. (1994) e Duncan et al. (1999), que consiste em minimizar a soma de quadrados dos desvios entre a proporção real dos alcanos (componentes da dieta) e as proporções calculadas nas fezes:

$$
Z=\sum_{i=1}^{N}\left(\frac{x \cdot H_{i . p}+(1-x) \cdot H_{i . q}}{x \cdot H_{\text {tot. } p}+(1-x) \cdot H_{\text {tot.q }}}-\frac{F_{i}}{F_{\text {tot }}}\right)^{2}
$$

em que $z$ é a soma de quadrados dos desvios; $x$, a proporção do componente $p$ na dieta; $H$ i.p, a concentração do $n$-alcano $i$ no componente $p$ na dieta; $H$ i.q, a concentração do $n$-alcano $i$ no componente $q$ na dieta; $F i$, a concentração do $n$-alcano $i$ nas fezes; $H$ tot.p, a soma das concentrações dos $n$-alcanos no componente $p$ na dieta; $H$ tot.q, a soma das concentrações dos $n$-alcanos no componente $q$ na dieta; F tot, a soma das concentrações dos n-alcanos nas fezes.

A minimização da soma dos quadrados dos desvios foi realizada por meio do programa computacional Microsoft Excel 2000, pelo procedimento Solver.

Os resultados foram submetidos à análise de variância utilizando-se os programas computacionais SAEG 5.0 (UFV) e SAS (1992).

As concentrações dos alcanos foram calculadas por análise de regressão, considerando os níveis de A. pintoi na dieta. Para comparar a média geral das concentrações dos alcanos, aplicou-se o teste Tukey $(\mathrm{P}<0,05)$.

O poder discriminatório dos $n$-alcanos foi determinado utilizando-se as variáveis canônicas pelas análises multivariadas.

Para análise da acurácia e precisão das estimativas de composição botânica, aplicou-se o teste $\mathrm{t}(\mathrm{P}<0,05)$, comparando as médias estimadas e considerando as diferenças das estimativas (proporção real subtraída da proporção estimada), o coeficiente de variação e o erro-padrão da média (Willians, 1993; Detmann et al., 2001; Vicente et al., 2002). 
O comportamento das observações da proporção real foi comparado ao comportamento estimado pela técnica, realizando-se um ajuste da regressão linear, obtendo-se uma equação da reta. Os coeficientes de regressão (b) e o intercepto da reta (a) foram testados se $b \neq 1$ e $a \neq 0$, pelo teste $t$ (Dove, 1992).

\section{Resultados e Discussão}

$\mathrm{Na}$ Tabela 1 constam os valores para as concentrações de $n$-alcanos $\mathrm{C}_{24}$ a $\mathrm{C}_{36}$ das dietas fornecidas aos animais. As extrações das amostras de fenos sem o padrão interno $\left(\mathrm{C}_{34}\right)$ comprovaram que o alcano $\mathrm{C}_{34}$ não foi identificado nas análises e, portanto, não existe necessidade de correção deste alcano das amostras com o uso do padrão interno.

Verificou-se predomínio dos alcanos de cadeias ímpares sobre os pares e que as concentrações dos alcanos de cadeia longa, principalmente o $\mathrm{C}_{31}$ e o $\mathrm{C}_{33}$, foram maiores $(\mathrm{P}<0,05)$ e com menores coeficientes de variação. A proporção de $A$. pintoi na dieta não influenciou $(\mathrm{P}>0,05)$ a concentração dos alcanos $\mathrm{C}_{24}, \mathrm{C}_{25}, \mathrm{C}_{26}, \mathrm{C}_{28}$ e $\mathrm{C}_{32}$, porém, teve efeito linear negativo $(\mathrm{P}<0,05)$ sobre a concentração total dos alcanos $\mathrm{C}_{27}, \mathrm{C}_{29}, \mathrm{C}_{30}, \mathrm{C}_{31}, \mathrm{C}_{33}, \mathrm{C}_{35}, \mathrm{C}_{36}$.

As concentrações superiores ou próximas a $50 \mathrm{mg} / \mathrm{kg}$ MS (Laredo et al., 1991; Chen et al., 1998) e distintas para as duas espécies de fenos indicam a possibilidade de discriminar os componentes da dieta fornecida aos ovinos. Havendo diferença no perfil de concentração dos $n$-alcanos entre as espécies, observa-se potencial de estimar a composição botânica das dietas (Dove \& Mayes, 1991; Newman et al., 1995; Dove et al., 1996, Côrtes et al., 2005), de modo que, quanto mais distinto o perfil de $n$-alcanos nas plantas mais acuradas as estimativas de composição botânica da dieta (Dove \& Mayes, 1999).

$\mathrm{Na}$ Tabela 2 constam as relações dos alcanos $\mathrm{C}_{29}: \mathrm{C}_{31}$, $\mathrm{C}_{29}: \mathrm{C}_{33} \mathrm{e}_{31}: \mathrm{C}_{33}$. As relações desses alcanos são menores que das escritas por Dove \& Mayes (1991) e Dove (1992) e podem ser atribuídas às baixas concentrações dos alcanos $\mathrm{C}_{29}$ e $\mathrm{C}_{31}$ nas espécies estudadas em relação a espécies forrageiras de clima temperado. $\mathrm{O}$ aumento da participação de $A$. pintoi na dieta acarretou maiores diferenças na concentração dos alcanos $\mathrm{C}_{29}: \mathrm{C}_{33}$ e $\mathrm{C}_{31}: \mathrm{C}_{33}$, refletindo a importância desses alcanos nos cálculos de composição.

Tabela 2 - Relação da concentração de alcanos na dieta de ovinos

Table 2 - $\quad$ Alkane concentration ratio on diet fed to sheep

\begin{tabular}{lccc}
\hline $\begin{array}{l}\text { Nível de A. pintoi } \\
\text { A. pintoi level }\end{array}$ & \multicolumn{3}{c}{$\begin{array}{c}\text { Relação } \\
\text { Ratio }\end{array}$} \\
\cline { 2 - 4 } & $\mathrm{C}_{29}: \mathrm{C}_{31}$ & $\mathrm{C}_{29}: \mathrm{C}_{33}$ & $\mathrm{C}_{31}: \mathrm{C}_{33}$ \\
\hline 0 & 0,362 & 0,196 & 0,541 \\
15 & 0,365 & 0,202 & 0,552 \\
30 & 0,369 & 0,209 & 0,566 \\
45 & 0,374 & 0,218 & 0,584 \\
60 & 0,380 & 0,230 & 0,607 \\
100 & 0,405 & 0,297 & 0,734 \\
\hline
\end{tabular}

Tabela 1 - Concentração de $n$-alcanos (mg/kgMS) em amostras de Brachiaria decumbens e Arachis pintoi e suas respectivas misturas fornecidas para ovinos

Table 1 - Concentration of $n$-alkanes ( $\quad$ Cof $/ \mathrm{kgDM})$ in samples of Brachiaria decumbens and Arachis pintoi and in the mixtures of both forages fed to sheep

\begin{tabular}{|c|c|c|c|c|c|c|c|c|c|}
\hline \multirow{3}{*}{$\begin{array}{l}N \text {-alcano }{ }^{1} \\
N \text {-alkane }\end{array}$} & \multirow{2}{*}{\multicolumn{6}{|c|}{$\begin{array}{c}\text { Nível de } A . \text { pintoi }(\%) \\
\text { A. pintoi level }\end{array}$}} & \multirow[t]{3}{*}{$\mathrm{CV}(\%)^{3}$} & \multirow{3}{*}{$\begin{array}{c}\text { Efeito }^{4} \\
\text { Effect }\end{array}$} & \multirow{3}{*}{$\begin{array}{c}\text { Média }^{5} \\
\text { Mean }\end{array}$} \\
\hline & & & & & & & & & \\
\hline & 0 & 15 & 30 & 45 & 60 & $100^{2}$ & & & \\
\hline $\mathrm{C}_{24}$ & 15,97 & 16,02 & 16,07 & 16,12 & 16,17 & 16,30 & 14,35 & $\mathrm{NS}$ & $16,11^{\mathrm{e}}$ \\
\hline $\mathrm{C}_{25}$ & 5,44 & 5,71 & 5,98 & 6,24 & 6,51 & 7,22 & 22,77 & NS & $6,18^{\mathrm{e}}$ \\
\hline $\mathrm{C}_{26}$ & 9,36 & 8,91 & 8,47 & 8,02 & 7,58 & 6,39 & 22,78 & NS & $8,12^{\mathrm{e}}$ \\
\hline $\mathrm{C}_{27}$ & 10,66 & 9,77 & 8,87 & 7,97 & 7,08 & 4,69 & 21,12 & LIN & $8,17^{\mathrm{e}}$ \\
\hline $\mathrm{C}_{28}$ & 75,13 & 68,00 & 60,87 & 53,74 & 46,62 & 27,60 & 34,02 & NS & $55,33^{c}$ \\
\hline $\mathrm{C}_{29}$ & 38,71 & 35,97 & 33,23 & 30,49 & 27,75 & 20,44 & 17,77 & LIN & $31,10^{\mathrm{de}}$ \\
\hline $\mathrm{C}_{30}$ & 10,67 & 9,73 & 8,79 & 7,85 & 6,91 & 4,41 & 10,74 & LIN & $8,06^{\mathrm{e}}$ \\
\hline $\mathrm{C}_{31}$ & 107,01 & 98,53 & 90,06 & 81,58 & 73,10 & 50,50 & 15,11 & LIN & $83,46^{b}$ \\
\hline $\mathrm{C}_{32}$ & 7,92 & 10,83 & 13,74 & 16,65 & 19,57 & 27,33 & 15,39 & NS & $16,01^{\mathrm{cde}}$ \\
\hline $\mathrm{C}_{33}$ & 197,86 & 178,50 & 159,15 & 139,80 & 120,45 & 68,84 & 9,89 & LIN & $144,10^{\mathrm{a}}$ \\
\hline $\mathrm{C}_{35}$ & 52,73 & 47,64 & 42,56 & 37,48 & 32,39 & 18,83 & 7,53 & LIN & $38,61^{\mathrm{cd}}$ \\
\hline $\mathrm{C}_{36}$ & 8,58 & 8,14 & 7,70 & 7,26 & 6,82 & 5,65 & 13,92 & LIN & $7,36^{\mathrm{e}}$ \\
\hline Total & 540,04 & 497,77 & 455,49 & 413,21 & 370,94 & 258,20 & 14,40 & LIN & 422,61 \\
\hline
\end{tabular}

${ }^{1}$ Alcano $\mathrm{C}_{34}$ não identificado nas amostras extraídas sem o padrão interno ( $C_{34}$ alkane was not identified in the extracted samples without the internal standard).

2 Não fornecida aos ovinos (not supplied to the animals).

$3 \mathrm{CV}(\%)=$ coeficiente de variação (coefficient of variation).

${ }^{4}$ Efeito (effect): LIN = Linear; NS - não-significativo (not significant).

${ }^{5}$ Médias seguidas de letras diferentes na coluna diferem $(P<0,05)$ pelo teste Tukey (means within a row followed by different letters are different $(P<0.05)$ by Tukey test). 
Na Tabela 3 são apresentados os coeficientes de ponderação dos $n$-alcanos na variável canônica. Para escolha dos $n$-alcanos nos cálculos da composição botânica utilizando a minimização da soma de quadrados dos desvios entre a proporção real dos alcanos (componentes da dieta) e as proporções calculadas nas fezes (Duncan et al., 1999), foram escolhidos os alcanos com maiores coeficientes de ponderação (em valores absolutos). Em seguida, iniciando pelo número mínimo de dois alcanos, foi adicionado um alcano de cada vez até que todos foram usados no cálculo da composição botânica. Os valores reais e estimados são apresentados na Tabela 4.

Tabela 3 - Coeficientes de ponderação dos $n$-alcanos na variável canônica

Table 3 - $\quad$ Coefficients showing the importance of the $n$-alkanes in the canonical variable

\begin{tabular}{lc}
\hline$N$-alcano & $\begin{array}{c}\text { Variável canônica } \\
\text { Canonical variable }\end{array}$ \\
\hline $\mathrm{C}_{35}$ & $-0,993$ \\
$\mathrm{C}_{33}$ & $-0,986$ \\
$\mathrm{C}_{30}$ & $-0,984$ \\
$\mathrm{C}_{31}$ & $-0,971$ \\
$\mathrm{C}_{27}$ & $-0,926$ \\
$\mathrm{C}_{29}$ & $-0,880$ \\
$\mathrm{C}_{36}$ & $-0,854$ \\
$\mathrm{C}_{26}$ & $-0,690$ \\
$\mathrm{C}_{25}$ & 0,687 \\
$\mathrm{C}_{28}$ & $-0,323$ \\
$\mathrm{C}_{24}$ & $-0,173$ \\
\hline
\end{tabular}

O uso das análises multivariadas para discriminar os alcanos de maior importância constitui um procedimento de grande valor para estimativa das proporções de A. pintoi na dieta (Côrtes et al., 2005a), por ordenar os alcanos com maior poder discriminatório. Entretanto, existe um número mínimo e máximo de alcanos que podem ser utilizados para estimar com acurácia e precisão a composição botânica.

$\mathrm{O}$ aumento do número de alcanos disponíveis oferece melhores estimativas e a possibilidade de se distinguir maior número de espécies ou frações dessas espécies, porém, nem todos os alcanos possuem poder para discriminar os componentes da dieta por adicionarem importantes fontes de erro às estimativas (Dove \& Mayes, 1999).

As técnicas de análises multivariadas avaliam simultaneamente um conjunto de características considerando as correlações existentes e permitindo que inferências sobre o conjunto de características sejam feitas em um nível de significância conhecido (Sakaguti et al., 1997). Desse modo, as análises multivariadas teriam grande importância em experimentos com número elevado de variáveis, por exemplo, quando a técnica de $n$-alcanos é utilizada para discriminar componentes de dietas (Côrtes et al., 2005a).

$\mathrm{Na}$ Tabela 4 são descritos os valores reais e estimados das proporções de A. pintoi da dieta dos animais. O uso de apenas dois $\left(\mathrm{C}_{35}\right.$ e $\left.\mathrm{C}_{33}\right)$ e três $\left(\mathrm{C}_{35}, \mathrm{C}_{33}\right.$ e $\left.\mathrm{C}_{30}\right)$ alcanos com maior poder discriminatório foi menos acurado $(\mathrm{P}<0,05)$ que o uso de número crescente dos alcanos nos cálculos, porém, a partir de três alcanos, as estimativas não diferiram

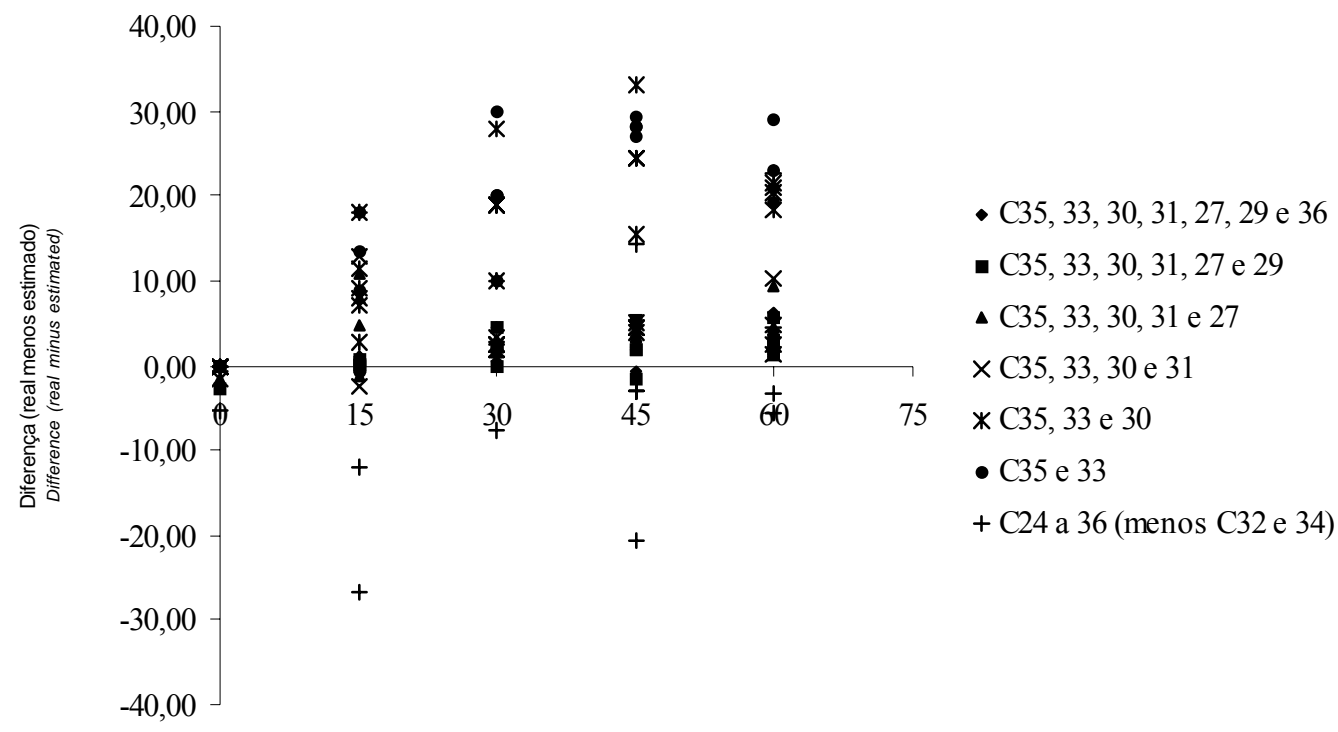

Proporção de A. pintoi

A. pintoi proportion

Figura 1 - Diferenças entre as proporções reais e estimadas de $A$. pintoi dos $n$-alcanos utilizados nos cálculos.

Figure 1 - Difference between the observed and estimated proportions of A. pintoi according to the $n$-alkanes used in the calculation. 
$(\mathrm{P}>0,05)$ entre si. No entanto, o uso dos sete primeiros alcanos mais discriminatórios (simultaneamente) foi mais preciso, pois apresentou menor coeficiente de variação. $\mathrm{O}$ uso de todos os alcanos $\left(\mathrm{C}_{24}\right.$ a $\left.\mathrm{C}_{36}\right)$ nos cálculos acarretou redução da precisão das estimativas, indicando um número mínimo e máximo de alcanos que melhor estime a composição botânica nas espécies utilizadas.

A acurácia e a precisão das estimativas podem ser mais bem demonstradas por meio do gráfico de dispersão entre o valor real e o estimado da proporção de $A$. pintoi (Figura 1). Os pontos mais dispersos e distantes do eixo das abscissas são estimativas utilizando números menores de alcanos nos cálculos. Verifica-se também que, com os níveis mais baixos de participação da leguminosa na dieta, as estimativas foram menos precisas (maior coeficiente de variação) que quando utilizado menor número de alcanos nos cálculos. O aumento da participação dos alcanos (discriminatórios) acarreta menores variações, mesmo quando a participação da leguminosa é baixa.

Em simulações de misturas com diferentes proporções de fenos de Arachis pintoi e Brachiaria brizantha, Côrtes et al. (2005b) observaram que, nos cálculos da composição, os alcanos $\mathrm{C}_{29}, \mathrm{C}_{31}, \mathrm{C}_{33}$ e $\mathrm{C}_{35}$ são de maior importância nas estimativas em relação a outras combinações aleatórias dos alcanos. Nesse trabalho, os autores observaram que a precisão das estimativas aumentava com a maior participação da leguminosa na amostra.

Constam na Tabela 5 as equações de regressão e seus parâmetros para os alcanos utilizados nas estimativas (valor real em relação ao estimado). O intercepto (a) das equações

Tabela 4 - Proporções real e estimada de Arachis pintoi na dieta ${ }^{1}$ Table 4 - Observed and estimated proportion of Arachis pintoi in the diet

\begin{tabular}{lccccccc}
\hline $\begin{array}{l}\text { Real } \\
\text { Observed }\end{array}$ & \multicolumn{5}{c}{$\begin{array}{c}\text { Proporção estimada de A. pintoi na dieta }(\%)^{3} \\
\text { Estimated proportion of A. pintoi in the diet }\end{array}$} \\
\cline { 2 - 7 } & 1 & 2 & 3 & 4 & 5 & 7 \\
\hline 0 & 0 & 0 & 0 & 0 & - & 0 \\
CV (\%) & - & - & - & - & - & 0 \\
15,00 & $5,14^{\mathrm{b}}$ & $7,43^{\mathrm{b}}$ & $5,89^{\mathrm{b}}$ & $9,42^{\mathrm{b}}$ & $14,78^{\mathrm{b}}$ & $14,17^{\mathrm{b}}$ & $30,59^{\mathrm{a}}$ \\
CV $(\%)$ & $(154,60)$ & $(48,85)$ & $(147,26)$ & $(54,57)$ & $(2,85)$ & $(1,25)$ & $(83,35)$ \\
30,00 & $10,00^{\mathrm{b}}$ & $11,02^{\mathrm{b}}$ & $27,42^{\mathrm{a}}$ & $27,66^{\mathrm{a}}$ & $27,83^{\mathrm{a}}$ & $27,58^{\mathrm{a}}$ & $28,89^{\mathrm{a}}$ \\
CV $(\%)$ & $(81,65)$ & $(66,55)$ & $(2,07)$ & $(1,89)$ & $(7,06)$ & $(6,14)$ & $(25,13)$ \\
45,00 & $16,76^{\mathrm{b}}$ & $20,70^{\mathrm{b}}$ & $40,63^{\mathrm{a}}$ & $41,62^{\mathrm{a}}$ & $43,13^{\mathrm{a}}$ & $43,05^{\mathrm{a}}$ & $48,07^{\mathrm{a}}$ \\
CV $(\%)$ & $(5,54)$ & $(35,13)$ & $(1,12)$ & $(1,08)$ & $(6,65)$ & $(5,26)$ & $(29,63)$ \\
60,00 & $36,93^{\mathrm{b}}$ & $39,74^{\mathrm{b}}$ & $55,33^{\mathrm{a}}$ & $55,49^{\mathrm{a}}$ & $56,94^{\mathrm{a}}$ & $56,41^{\mathrm{a}}$ & $55,49^{\mathrm{a}}$ \\
CV $(\%)$ & $(11,48)$ & $(3,70)$ & $(7,14)$ & $(6,32)$ & $(3,20)$ & $(3,65)$ & $(23,26)$
\end{tabular}

${ }^{1}$ Médias seguidas de letras diferentes na linha diferem $(P<0,05)$ pelo teste $t(M e a n s$ within a row followed by different letters differ $[P<0.05]$ by $t$ test).

$2 \mathrm{CV}(\%)=$ coeficiente de variação (coefficient of variation).

$31=\mathrm{C}_{35}, \mathrm{C}_{33}$

$2=C_{35}, C_{33}, C_{30}$

$3=\mathrm{C}_{35}, \mathrm{C}_{33}, \mathrm{C}_{30}, \mathrm{C}_{31}$.

$4=\mathrm{C}_{35}, \mathrm{C}_{33}, \mathrm{C}_{30}, \mathrm{C}_{31}, \mathrm{C}_{27}$

$5=\mathrm{C}_{35}, \mathrm{C}_{33}, \mathrm{C}_{30}, \mathrm{C}_{31}, \mathrm{C}_{27}, \mathrm{C}_{29}$

$6=\mathrm{C}_{35}, \mathrm{C}_{33}, \mathrm{C}_{30}, \mathrm{C}_{31}, \mathrm{C}_{27}, \mathrm{C}_{29}, \mathrm{C}_{36}$.
7 = Todos os alcanos (menos $\mathrm{C}_{32}$ e $\mathrm{C}_{34}$ ) (All alkanes with the exception of $C_{32}$ and $C_{34}$ ).

Tabela 5 - Equações de regressão, interceptos da equação (a), coeficientes de regressão (b) e coeficientes de correlação (r) para estimativa das proporções de $A$. pintoi na dieta

Table 5 - Regression equation, intercept of the equation (a), regression coefficients (b) and correlation coefficients ( $r$ ) for the estimation of the proportions of $A$. pintoi in the diet

\begin{tabular}{|c|c|c|c|c|c|}
\hline $\begin{array}{l}N \text {-alcano } \\
N \text {-alkane }\end{array}$ & $\begin{array}{l}\text { Regressão } \\
\text { Regression }\end{array}$ & $\mathrm{R}^{2}$ & $a \neq 0$ & $b \neq 1$ & $\mathrm{r}^{*}$ \\
\hline (1) $\mathrm{C} 35,33$ & $\hat{Y}=-2,13276+0,549931 X$ & 0,7639 & NS & $* *$ & 0,8740 \\
\hline (2) $\mathrm{C} 35,33$ e 30 & $\hat{Y}=-1,57292+0,598293 X$ & 0,8151 & NS & $* *$ & 0,9028 \\
\hline (3) $\mathrm{C} 35,33,30$ e 31 & $\hat{\mathrm{Y}}=-1,80571+0,944469 \mathrm{X}$ & 0,9586 & NS & NS & 0,9791 \\
\hline (4) $\mathrm{C} 35,33,30,31$ e 27 & $\hat{Y}=-0,37791+0,929573 X$ & 0,9839 & NS & NS & 0,9919 \\
\hline (5) $\mathrm{C} 35,33,30,31,27$ e 29 & $\hat{\mathrm{Y}}=1,7030+0,91903 \mathrm{X}$ & 0,9888 & NS & NS & 0,9944 \\
\hline (6) $\mathrm{C} 35,33,30,31,27,29$ e 36 & $\hat{\mathrm{Y}}=1,42496+0,917549 \mathrm{X}$ & 0,9912 & NS & NS & 0,9956 \\
\hline (7) $\mathrm{C} 24$ a $\mathrm{C} 36$ & $\hat{\mathrm{Y}}=8,90695+0,818803 \mathrm{X}$ & 0,5888 & $* *$ & NS & 0,7674 \\
\hline
\end{tabular}

* Significativo $P<0,01$ (significant at $P<0.01$ ).

** Significativo $(P<0,05)$ (significant at $P<0.05)$

NS = não-significativo (not significant). 
para proporção de $A$. pintoi na dieta não diferiu $(\mathrm{P}>0,05)$ de zero $(a=0)$, pelo teste $t$, para as estimativas utilizando os alcanos dentro da variável canônica, porém, com o uso de todos os alcanos $\left(\mathrm{C}_{24}, \mathrm{C}_{36}\right)$, o intercepto (a) da equação foi diferente $(\mathrm{P}<0,05)$ de zero $(\mathrm{a} \neq 0)$. Para o coeficiente de regressão (b), apenas as estimativas utilizando os alcanos $\mathrm{C}_{35}$ a $_{33}$ e $_{35}$ a $\mathrm{C}_{30}$ foram diferentes $(\mathrm{P}<0,05)$ de $1(\mathrm{~b} \neq 1)$ pelo teste $\mathrm{t}$.

Para as equações 3 a 6 , nas quais o intercepto foi igual a zero e o coeficiente de regressão não diferiu de 1, pode-se afirmar que os métodos foram equivalentes, pois os valores estimados foram semelhantes aos observados.
Na equação 7 (uso de todos os alcanos), existe uma correspondência entre o real e estimado, porém com a necessidade de um fator de correção constante, no caso a estimativa do intercepto da equação. Entretanto, a falta de ajuste da equação $\left(R^{2}=0,5888\right)$ pode acarretar correção pouco precisa.

Nas equações 1 e 2, existe um vício global de estimação $(b \neq 1)$. Essas equações representam comportamento diferenciado entre a proporção real e a estimada.

Entre as equações em que os métodos foram equivalentes (real com estimado), a equação 6 (utilizando todos os alcanos da variável canônica) foi a mais acurada nas
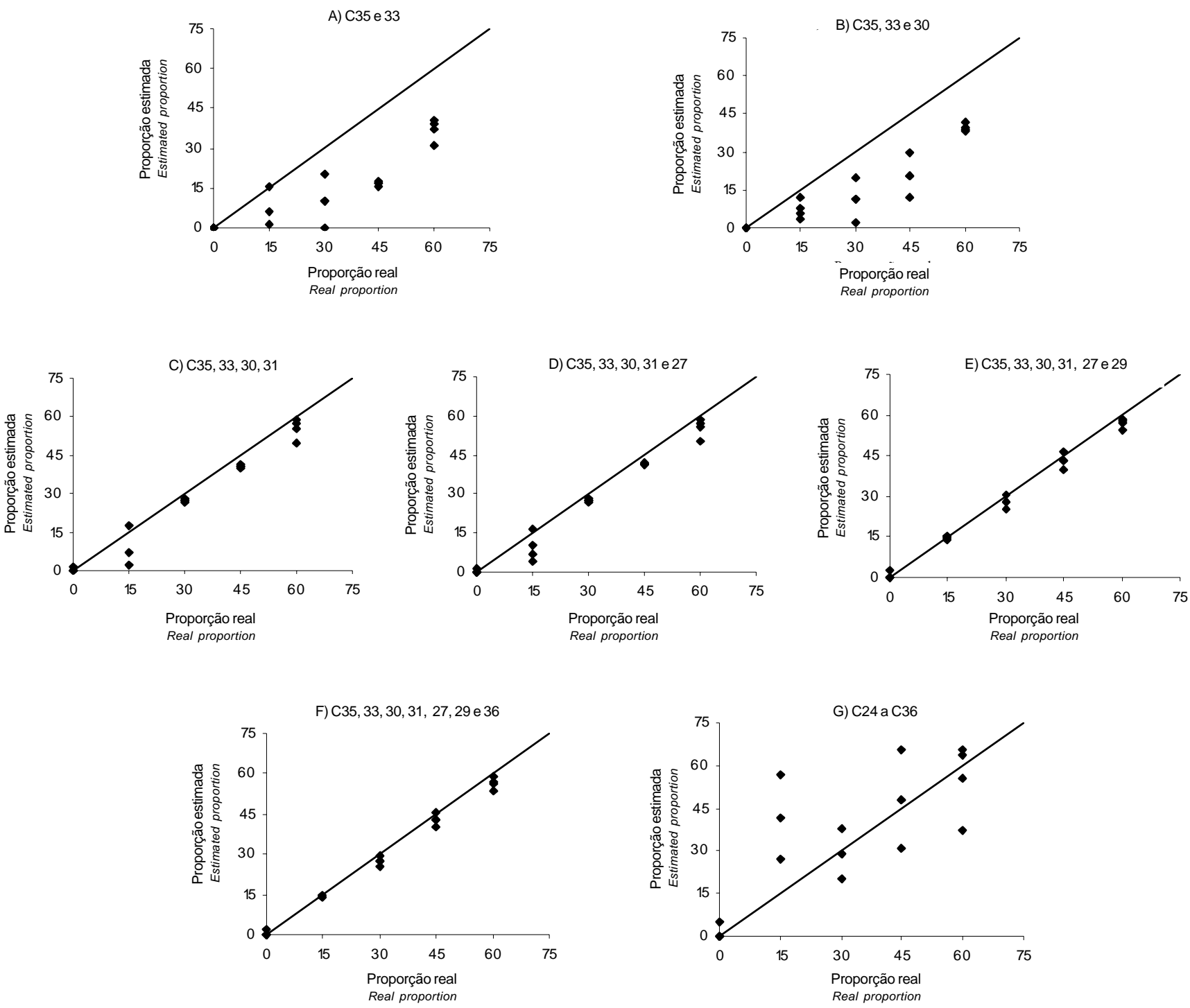

Figura 2 - Relação entre as proporções reais consumidas por ovinos e estimadas pelos $n$-alcanos de Arachis pintoi de acordo com os $n$-alcanos utilizados nos cálculos.

Figure 2 - Relationship between observed and estimated proportions of Arachis pintoi $n$-alkanes used to estimate intake in sheep according to the $n$-alkanes used in the calculations. 
estimativas por ter apresentado ajuste da equação pouco maior que os demais. A correlação também foi crescente para o acréscimo do alcano nos cálculos; a equação 6 apresentou a melhor correlação real com estimado.

Na Figura 2 são representados os gráficos de dispersão das estimativas de proporção de A. pintoi na dieta utilizando-se diferentes números de alcanos nos cálculos.

As melhores estimativas, como descrito anteriormente, foram obtidas quando utilizados os alcanos $\mathrm{C}_{35}, \mathrm{C}_{33}, \mathrm{C}_{30}$, $\mathrm{C}_{31}, \mathrm{C}_{27}$ e $\mathrm{C}_{29}$ (Figura 2 - E) e $\mathrm{C}_{35}, \mathrm{C}_{33}, \mathrm{C}_{30}, \mathrm{C}_{31}, \mathrm{C}_{27}, \mathrm{C}_{29}$ $\mathrm{e}_{36}$ (Figura 2-F). Nas demais figuras, utilizando os outros alcanos nos cálculos, os valores estimados foram bastante dispersos e subestimados (pontos abaixo da reta na maioria dos casos).

Em geral, as técnicas utilizando $n$-alcanos são eficientes para predizer a composição de dietas em situações de simulação, quando se conhece a dieta dos animais, contudo, quando a técnica é transferida para situações de campo, ainda são detectados problemas nas estimações. Desse modo, é fundamental experimentar métodos matemáticos que selecionem os alcanos a ser utilizados objetivando minimizar o erro das estimativas.

\section{Conclusões}

Os $n$-alcanos possibilitam estimativas precisas e acuradas com as espécies forrageiras estudadas. As análises multivariadas representam alternativas de cálculo para melhorar a aplicabilidade da técnica de $n$-alcanos na discriminação de dietas para ruminantes. Estudos envolvendo estimativas da composição botânica com número maior de componentes ou frações das plantas na dieta dos animais sob pastejo, em condições tropicais, são importantes para melhorar a aplicabilidade da técnica.

\section{Literatura Citada}

CHEN, W.; LEFROY, R.D.B.; SCOTT, J.M. et al. Field variations in alkane signatures among plant species in 'degraded' and perennial pastures on the Northern Tablelands of New South Wales. Australian Journal of Agricultural Research, v.49, n. 8, p.263, 1998 .

CHEN, W.; SCOTT, J.M; BLAIR, G.J. et al. Using plant cuticular alkanes to study plant-animal iteration on pastures. Canadian Journal of Animal Science, v.79, n.4, p.553-556, 1999.

CORTES, C.; DAMASCENO, J.C.; BECHET, G. et al. Species composition in ryegrass (Lolium perenne)/tall fescue (Festuca arundinacea) mixtures using various combinations of n-alkanes. Grass and Forage Science, v.60, p.254-261, 2005.

CÔRTES, C.; DAMASCENO, J.C.; FUKUMOTO, N.M. et al. Potencial discriminatório dos $n$-alcanos em plantas forrageiras tropicais por análises multivariadas. Revista Brasileira de Zootecnia, v.34, n.4, p.1079-1087, 2005a.
CÔRTES, C.; DAMASCENO, J.C.; PAINE, R.C. et al. Uso de n-alcanos na estimativa da composição botânica em amostras com diferentes proporções de Brachiaria brizantha e Arachis pintoi. Revista Brasileira de Zootecnia, v.34, n.5, p.1468-1474, $2005 b$

DETMANN, E.; PAULINO, M.F.; ZERVOUDAKIS, J.T. et al. Cromo e indicadores internos na determinação do consumo de novilhos mestiços, suplementados, a pasto. Revista Brasileira de Zootecnia, v. 30, n.5, p.1600-1609, 2001.

DOVE, H. Using the $n$-alkanes of plant cuticular wax to estimate the species composition of herbage mixtures. Australian Journal of Agricultural Research, v.43, p.1711-1724, 1992.

DOVE, H.; MAYES, R.W. Markers for estimation in herbivores. In: INTERNATIONAL SYMPOSIUM ON THE NUTRITION OF Herbivores, 5., 1999, Texas. Proceedings... Texas: Proceedings of satellite symposium, 1999. (In press).

DOVE, H.; MAYES, R.W. Plant wax components: A new approach to estimating intake and diet composition in herbivores American Institute of Nutrition, v.126, p.13-26, 1996.

DOVE, H.; MAYES, R.W. The use of plant wax alkanes as marker substances in studies of the nutrition of herbivores: a review. Australian Journal of Agricultural Research, v.42, p.913952, 1991

DOVE, H.; MAYES, R.W.; FREER, M. Effects of species, plant part, and plant age on the $n$-alkanes concentration in the cuticular wax of pasture plants. Australian Journal of Agricultural Research, v.47, p.1333-1347, 1996.

DUNCAN, A.J.; MAYES, R.W.; LAMB, C.S. et al. The use of naturally occurring and articially applied n-alkanes as markers for estimation of short-term diet composition and intake in sheep. Journal Agriculture Science, v.132, p.233-246, 1999.

LAREDO, M.A.; SIMPSON, D.J.; ORPIN, C.G. The potential for using $n$-alkanes in tropical forages as a marker for the determination of dry matter by grazing ruminants. Journal of Agriculture Science, v.117, p.355-361, 1991.

MAYES, R.W.; BERESFORD, N.A.; LAMB, C.S. et al. Novel approaches to the estimation of intake and bioavailability of radiocaesium in ruminants grazing forested areas. Science of the Total Environment, v.157, p.289-300, 1994.

NEWMAN, J.A.; THOMPSON, W.A.; PENNING, P.D. Least-square estimation of diet composition from $n$-alkanes in herbage and faces using matrix mathematics. Australian Journal of Agricultural Research, v.46, p.793-805, 1995.

SAKAGUTI, E.S.; SILVA, M.A.; REGAZZI, A.J. et al. Análises multivariadas em um dialelo completo e desbalanceado com coelhos das raças Nova Zelândia Branco, Califórnia e Chinchila Revista Brasileira de Zootecnia, v.26, n.3, p.501-507, 1997.

UNIVERSIDADE FEDERAL DE VIÇOSA - UFV. Sistema para Análise Estatísticas e Genéticas - SAEG. Viçosa, MG: 1993. (CD-ROM).

STATISTICAL ANALYSES SYSTEM - SAS. SAS/STAT: changes and enhancements. Release 6.07. Cary: 1992. (Technical Report, P-229).

VICENTE, P.M.; DAMASCENO, J.C.; SANTOS, G.T. et al. Efeito de estratégia de suplementação com concentrado no desempenho de cabras mestiças Saanen, em dois sistemas de produção. Revista Brasileira de Zootecnia, v31, n.1, p.460-466, 2002 (sup1.).

VULICH, S.A.; HANRAHAN, J.P.; CROWLEY, B.A. Modification of the analytical procedures for the determination of herbage and fecal n-alkanes used in the estimation of herbage intake. Journal of Agricultural Science, v.124, p.71-77, 1995

WILLIANS, J.C. An empirical model for the lactation curve of white British dairy goats. Animal Production, v.57, p.91-97, 1993. 